\title{
Dynamic Frequency Hopping in Cellular Fixed Relay Networks
}

\author{
Omer Mubarek, Halim Yanikomeroglu \\ Broadband Communications \& Wireless Systems Centre \\ Carleton University, Ottawa, Canada \\ \{mubarek, halim\}@sce.carleton.ca
}

\author{
Shalini Periyalwar \\ Wireless Technology Labs \\ Nortel Networks, Ottawa, Canada \\ speriyal@nortelnetworks.com
}

\begin{abstract}
This paper proposes a new interference management/avoidance technique for cellular fixed relay networks with aggressive channel reuse: Dynamic Frequency Hopping with Limited Information (DFH-LI). It incorporates relaying with a combination of dynamic frequency hopping (DFH) and random frequency hopping (RFH). This technique results in enhanced high data rate coverage and decreased outage.
\end{abstract}

Keywords-component; Relaying, mesh networks, multihop networks, frequency hopping, resource allocation

\section{INTRODUCTION}

Significant effort has been put on the search of costefficient high data rate coverage enhancement techniques in modern wireless communications systems; cell splitting, sectorized cells and smart antennas can be shown among these techniques. With the evolution of signal processing techniques in the recent years, especially smart antenna technologies have become popular (such as MIMO, V-BLAST and adaptive antennas). However, none of these techniques seem to be sufficient to enable almost-ubiquitous coverage in an economical way at the data rates envisaged for beyond-3G systems [1].

The system proposed in this paper assumes a broadband cellular mobile network with an aggressive channel reuse of more than 1 (up to 7); that is, a channel can be reused many times in the service region of a base station (BS). In order to deal with the striking interference problem of such aggressive channel reuse schemes, certain interference management/avoidance techniques have been proposed [2]. These techniques require transmission coordination of BSs, which are interferers of each other. However, in the current wireless communications architecture, neighboring BSs do not have a wired link between each other. Therefore, information exchange, hence transmission coordination cannot be done in a timely manner among BSs.

Integrating relaying concept into conventional wireless communications systems increases high data rate coverage in a cost-effective manner while decreasing outage, among many other benefits [1]. This paper proposes a novel resource allocation scheme by introducing frequency hopping $(\mathrm{FH})$ in a cellular fixed relay network.

Recently AT\&T and WINLAB proposed a new interference management technique called Dynamic Frequency Hopping (DFH) [3]. DFH incorporates a nontraditional Dynamic Channel Allocation (DCA) scheme with slow FH. The main objective is to provide capacity improvements through the addition of interference avoidance,

This work was supported by Wireless Tech. Labs, Nortel Networks, Ottawa which are higher than those provided by conventional $\mathrm{FH}$, while preserving interference averaging characteristics of conventional $\mathrm{FH}$ in order to provide robustness to changes in interference. Although DFH increases the performance compared to conventional systems as well as systems using random frequency hopping (RFH), it requires BS coordination. Thus, exploiting dynamic frequency hopping in current conventional wireless communications systems encounters the same practicality bottleneck described above. Therefore in our research we use DFH as a performance upper-bound.

In cellular fixed relay networks, BS and its relay stations (RSs) have a master-slave relation. In this architecture (wireless) links between a BS and its RSs already exist. Therefore, interference management can be facilitated through transmission coordination in a cell. This paper proposes a novel and practical interference management technique for cellular relay networks with very dense channel reuse.

\section{SYSTEM DESCRIPTION}

The proposed system architecture, Cellular Relay Network (CRN) is given in Fig. 1. The performance of this network will be compared with the conventional cellular network (CCN), which is the conventional architecture without relays. Due to the fact that downlink (DL) communication requires much higher speeds than uplink (UL), only DL performance will be analyzed [4].

The proposed two-hop cellular system is composed of one BS and six RSs placed uniformly around the BS. The RSs are deployed in the cell such that they can support a good link with the BS (most preferably at line-of-sight locations). Because of these RSs, each cell consists of seven neighborhoods, called subcells. Six of these subcells correspond to the RS neighborhoods and these subcells surround the BS neighborhood, which is the seventh and the center subcell.

The frequency (in this paper, a channel refers to a frequency channel without loss of generality) reuse in a neighborhood in a cell is up to one. This means that at a specific time the same frequency can be used up to seven times in a cell (since a cell consists of seven subcells or neighborhoods). The FH patterns assigned to user equipments (UEs) in a subcell are orthogonal to each other. Hence, there is no in-subcell interference. It is also assumed that there is no adjacent channel interference either. The only interference experienced by a UE is the out-of-subcell interference created by the RSs and/or BSs of the surrounding subcells. 
The BS - RS link is very important for the speed and quality of the end-to-end communication. Each RS serves to a number of UEs. The active UEs in the service region of a RS, receive all their data through that RS. Compared to the RS-UE or UE-BS link, the RS-BS link carries much more information. Therefore increasing the quality of this link increases the speed and the quality of the whole network. Thus, six directional antennas are assumed to be deployed between each BS and its RSs (one directional antenna for each RS-BS link). In addition, a BS and its six RSs use omnidirectional antennas to communicate with the UEs. UEs also use omni-directional antennas for communicating with the BS as well as with the RSs.

BSs operate in two different transmit power modes: high power mode and low power mode. Therefore a time-division multiplexing (TDM) scheme is used, where the time is divided into two time slots. Since RSs cannot transmit and receive at the same channel [1], these operations need to be performed in different time slots, too.

\section{A. Time Slot 1}

In this time slot, the BS transmits with high power (20 $\mathrm{W})$. It sends data to RSs while sending control and signaling messages to UEs; the high power mode enables the BS to reach the UEs at the periphery of the cell as well. This paper assumes that in the first time slot the BS does not transmit data to any UE (although it is an option).

Since RSs have good links (preferably line-of-sight) augmented by directional antennas with their serving BS, communication in this time slot is assumed to be perfect for all practical reasons. The focus of this paper is on the second time slot.

\section{B. $\quad$ Time Slot 2}

In the second time slot, the BS transmits at low power (3 $\mathrm{W})$, which is equal to the transmission power of the RSs. Therefore, there exists no dominant power source throughout the whole system (slot-level synchronization is assumed in the network). In this time slot, the RSs do not receive from the $\mathrm{BS}$; rather they transmit to the UEs in their coverage region.

In this proposed cellular relay network architecture, the interference to a UE in a certain neighborhood will come from the surrounding RSs and BSs; note that some of these interferers are out-of-cell interferers. Now DFH can be modified and applied to CRN. This modified technique is called Dynamic Frequency Hopping with Limited Information (DFH-LI), which will be explained in the next section.

\section{DYNAMIC FREQUENCY HOPPING WITH LIMITED INFORMATION (DFH-LI)}

This technique uses the basic principles of DFH to utilize the resources in the second time slot. DFH combines the advantages of both dynamic resource allocation (interference avoidance), and of FH (frequency diversity) [3]. FH will help in minimizing the effect of interference on the performance. The hopping patterns are going to be generated for active users on the fly, according to some measurements and calculations performed in real-time; i.e., there is no pre-defined FH pattern such as pseudo-random patterns or cyclic patterns.

The following two cases are investigated:

i. BS - UE communication

ii. RS - UE communication

In the second time slot, neither the BS nor the RSs perform control and signaling functions. In the analysis below, only the first subcell-tier of transmitters (only RSs in this case) will be considered as interferers. The analysis for two subcelltiers (RSs and BSs) of interferers is similar.

\section{A. $\quad B S-U E$ Communication}

The BS will assign a FH pattern to its users in its neighborhood. The serving BS should do this assignment according to a performance criterion such as the SINR (signalto-interference-plus-noise ratio) value. During pattern updates, the defective frequencies with SINR levels below the threshold SINR will be replaced with the frequencies supporting SINR levels above the threshold.

The crucial information the BS needs is the interference at the UE in a certain time slot with a certain FH pattern. The potential interferers are the six RSs surrounding the BS. In order for the BS to calculate the interference caused by these RSs at the UE, the BS needs to know:

i. Transmit power of the RSs,

ii. Resource utilization information of the RSs,

iii. Pathloss of the RSs to the UE.

Since in the first time slot the BS has assigned the resources to the RSs, and since the RSs have a constant transmission power, the BS already has the information i) and ii). The third piece of information comes from the UEs. Each UE measures the pathloss to the neighboring RSs, and its serving BS and reports this information to its serving BS (Fig. 2).

Having collected this information, the BS calculates the interference level at each available resource, determines the least interfered time slot and FH pattern pair, and assigns this to the UE. After this, the BS continuously monitors each user's performance and reassigns another resource if the performance (SINR in this case) degrades below the threshold level in a certain FH time slot.

\section{B. RS-UE Communication}

In the case of the RS communicating with one of its UEs, there are again six potential interferers: the serving BS of the cell, two RSs from the same cell and three RSs from different cells. The serving BS will assign the resources to the UE (Fig. 3).

In this paper a decentralized system is assumed, where communication and data transfer between different cells is minimized. Since three of the interferers are in different cells, DFH as proposed in [3], would require BSs of different cells to communicate with each other. In DFH-LI, on the other hand, the FH patterns are determined based on local information; that is, DFH-LI does not require inter-BS communication for FH pattern determination (obviously, the performance of DFH-LI will be inferior to that of DFH; but as 
it will demonstrated in Section $\mathrm{V}$, the difference is often small).

A UE performs pathloss measurements to the two in-cell RSs, the BS, and its serving RS, and sends this information to its RS. The RS passes this information to its serving BS, which will assign the FH patterns to the UE. According to $\mathrm{DFH}$, all the interferers need to report their transmission power level and resource utilization information (which $\mathrm{FH}$ pattern they are using in which time slot and at which power) to the BS which is going to assign resources to the UE [5]. However, the two in-cell RSs do not need to report their resource utilization information to the BS since from the previous time slot the $\mathrm{BS}$ has this resource utilization information (in the first time slot the BS determines which resources the RSs are going to use, and it keeps this information for the calculations in the second time slot). Thereby the serving BS has the necessary information for the serving RS as well as for the in-cell interferers. However, there is no communication between the three RSs in the other cells and the serving BS.

At this point the serving BS knows (with even partial information) with certainty at which frequencies in a time slot the SINR will be below the threshold value, so it does not assign these frequencies to the UE. These frequencies are blocked for that very frequency hop. Up to this part the operation is similar to DFH. However, since the BS is missing necessary interference information from other three out-of-cell $\mathrm{RSs}$, it does not know the quality level at the rest of the frequencies in that hop. Therefore, it can assign any of the frequencies which is not blocked by the DFH part of the FH scheme. However, there is no guarantee that the quality of service (QoS) will be at an acceptable level at that frequency. BS does not know whether the out-of-cell interfering RSs are using those frequencies or not, and if they are, how much interference they cause. Therefore, this proposed technique is called DFH with Limited Information.

\section{SimUlation MODEL}

In the simulation model log-normal shadowing with $0-\mathrm{dB}$ mean and 8-dB standard deviation is assumed. RF carrier frequency is $2 \mathrm{GHz}$ and transmission bandwidth is $50 \mathrm{MHz}$. Thermal noise with a noise figure of $8 \mathrm{~dB}$ is assumed. Isotropic antennas with unity gain are used at BSs, RSs, and UEs (in the second time slot that we investigate). Multipath fading is assumed to be taken care of by other means (such as antenna diversity). Adaptive modulation and coding is used where an appropriate modulation and coding level between half-rate QPSK and full-rate 64-QAM is determined based on the SINR value.

The performance of a UE is monitored over 192 time slots ${ }^{*}$. Assuming each six time slots makes a frame, 32 frames are considered in the simulations. The performance of the system is measured through average user spectral efficiency

\footnotetext{
* These time slots refer to different frequency hops and are different than the two time slots of the TDM scheme defined in the previous section. In the simulations, we are focusing on the second time slot only, where the BS has low power.
}

$(\mathrm{SE})^{ \pm}$, and outage probability. For systems not exploiting any kind of frequency hopping, a user has a certain SE and it experiences outage if its SINR is less than a certain threshold value $(4 \mathrm{~dB}$ in this paper which corresponds to half-rate QPSK). Therefore the outage probability is either $0 \%$ or $100 \%$ for one UE. For systems using a type of frequency hopping (RFH, DFH or DFH-LI), on the other hand, SE is given as the average SE of the 192 time slots. Outage probability is the ratio of the number of frames the UE is in outage over the total number of frames (which is 32). The UE is considered to experience outage in a certain frame if in all the six time slots of that frame its SINR is less than the threshold value of 4.0 $\mathrm{dB}$. Hence outage probability takes values between $0 \%$ and $100 \%$.

It is enough for a frame to have at least one time slot with SINR above the threshold in order not to experience outage. It is assumed that the information for the rest of the poor hops can be recovered using automatic repeat request (ARQ). However, if all the time slots are poor, then it means that there is no link for ARQ either, hence the frame experiences outage.

\section{Simulation RESUlts}

The objective of the simulations is to determine the improvement in high data rate coverage (through a potential increase in average spectral efficiency and a decrease in outage performance) for various loading ${ }^{\ddagger}$ levels by exploiting relay stations and using various frequency hopping techniques. These systems will be compared to the single hop systems exploiting and not exploiting the respective frequency hopping techniques.

The average user spectral efficiency versus loading plots for the two networks (CCN and $\mathrm{CRN}$ ) are shown in Fig. 4; in this figure the performances of these networks without $\mathrm{FH}$ (noFH), with RFH, with DFH and with DFH-LI (only the CRN network) cases have been compared.

In none of the networks, the introduction of RFH affects the average user spectral efficiency noticeably, due to the fact that RFH only averages the present interference. After applying RFH, interference experienced by high SINR users will increase (SE will decrease), while that experienced by poor SINR users will decrease (SE will increase). However, the total interference of the system will remain the same. Therefore the total and average SE of the system will be very similar to that of noFH system.

Another interesting result of Fig. 4 is the improvement in the average user SE and hence in the high data rate coverage with the introduction of relays. Although for low loading the difference in SE is not much (for loading $=0.1, \mathrm{CRN}-$ noFH improves SE by $66 \%$ over CCN-noFH), as the cells get busier, the results show that with the help of the relays CRN deals better with heavy traffic (for loading $=1.0, \mathrm{CRN}-\mathrm{noFH}$ improves SE by 203\%). Comparing CRN systems among each

\footnotetext{
${ }^{ \pm}$The SE values presented in the simulations belong to the second time slot only and are not the composite SE values of the two hops.

$¥$ Loading is defined as the ratio of users to the number of available frequencies in a cell. For CCN, we let loading vary between 0 and 1 , and for CRN between 0 and 7 .
} 
other, CRN-noFH and CRN-RFH perform the same while CRN-DFH and CRN-DFH-LI outperform noFH and RFH systems. At loading $=4$, the average SE for DFH-LI is $24 \%$ higher than that of noFH and RFH.

Fig. 5 shows the SE distribution for DFH-LI at various loading values. At low loading, there is not much interference in the system therefore high spectral efficiency users are in majority. For full loading, it is the opposite; DFH-LI cannot operate (since there are no spare frequencies), and this time users with poor SE are in majority. For intermediate loading, DFH-LI uses its interference averaging and avoidance features perfectly and there is a close to uniform distribution of users over all the SE values.

Fig. 6 presents the results of the outage analysis. It is clearly seen that introduction of relays decrease the outage probability of the system (at loading $=1.0$, for noFH case, outage probability drops from 0.56 to 0.08 ). When various $\mathrm{FH}$ techniques are applied, outage probability decreases even more (at loading = 4, CRN-noFH: 0.3, CRN-RFH: 0.08, CRNDFH and CRN-DFH-LI: 0.01). An interesting result is that outage probability remains 0 until loading $=1.0$ for CRN-RFH and until loading $=4.0$ for CRN-DFH/CRN-DFH-LI, while it is never 0 for $\mathrm{CRN}$-noFH.

\section{CONCLUSIONS AND FUTURE WORK}

In order to facilitate aggressive channel reuse, several interference management/avoidance techniques have been proposed in the literature which require BS coordination. However, since the current network architecture does not allow timely information exchange among neighbouring BSs, the proposed techniques have found only limited applications so far. In this context, this paper proposes a technique that we called "Dynamic Frequency Hopping with Limited Information" (DFH-LI), derived from AT\&T and WINLAB's $\mathrm{DFH}$, in cellular fixed relay networks. With this interference management/avoidance technique, the same channel can be reused up to seven times in each cell (via the integration of RSs) boosting the cell capacity/throughput. Having analyzed the performance of DFH-LI and compared it with various techniques we have come to the following conclusions.

Integrating various $\mathrm{FH}$ techniques and relays with the original CCN network improves average user spectral efficiency and outage probability, hence high data rate coverage. We consistently observe that being a hybrid $\mathrm{FH}$ technique of RFH and DFH, the performance of DFH-LI is a lot closer to DFH than it is to RFH. Combining this result with the fact that DFH-LI is proposed as a practical alternative to DFH makes DFH-LI very attractive for implementation in future wireless systems.

RFH decreases the outage by averaging the total interference throughout the whole system. Thereby, users with poor SINR increase their SINR above the threshold and do not experience outage (while SINR of high performance users decreases but still stays above the threshold). DFH and DFHLI take this one step further by adding interference avoidance to interference averaging. Therefore performance improvements for the DFH and DFH-LI cases are more prominent for both single hop and especially relay systems.

At full loading (loading $=1.0$ for $\mathrm{CCN}$ and loading $=7.0$ for CRN), the average SE and outage probability values converge for noFH and various FH schemes in each network. This result is due to the fact that $\mathrm{FH}$ techniques improve the system performance via spare frequencies. At full loading all the channels will be used in the interfering cells, and therefore the FH methods will lose their meaning. Users will experience a constant interference at every frequency regardless of whether a certain FH technique is exploited or not. This can be seen clearly in Fig. 4 and Fig. 6 (separately for $\mathrm{CCN}$ and CRN).

When the same FH systems are compared (noFH, RFH, DFH and DFH-LI) with each other for CCN and CRN networks separately it is observed that the systems using any FH technique are more robust to user traffic than the those not noFH systems; their performance does not degrade as fast as noFH due to their interference management/avoidance capabilities.

This paper raises a number of interesting issues and ideas for further investigation:

- Analyzing the performance of various frequency management/avoidance techniques for $\mathrm{CRN}$ in the presence of user mobility.

- Introducing frequency selective fading to the channel model.

- Performing the same analysis for the uplink.

- Considering a multi-hop cellular network with more than two hops. In this case, there would be some complications, such as dividing the time into more than two time slots, and considering efficient routing algorithms. An issue is where to deploy the relays; should the location of RSs satisfy a good BS-RS link or a good RS-RS link among neighbouring ring relays. The pathloss information coming from UEs far away from the BS will come over multiple hops to the BS; therefore delay will play a more important role on the efficiency of the system.

\section{REFERENCES}

[1] H. Yanikomeroglu, "Fixed and mobile relaying technologies for cellular networks," Second Workshop on Applications and Services in Wireless Networks (ASWN'02), pp. 75-81, 3-5 July 2002, Paris, France

[2] M. Rahman, Adaptive Modulation and Coding-Based Packet Scheduling with Inter-Base Station Coordination in Fixed Cellular Broadband Wireless Networks, M.A.Sc. Thesis, Supervisors: Prof. H. Yanikomeroglu and Prof. S. Mahmoud, Carleton University, May 2004 (available http://www.sce.carleton.ca/faculty/yanikomeroglu/Pub/rahman_thesis.pd f)

[3] Z. Kostic and N. Sollenberger, "Performance and Implementation of dynamic frequency hopping in limited-bandwidth cellular systems," IEEE Transactions on Wireless Communications, vol. 1, no. 1, pp.26-38, January, 2002.

[4] O. Mubarek, Dynamic Frequency Hopping in Cellular Fixed Relay Networks, M.A.Sc. Thesis, Supervisors: Prof. H. Yanikomeroglu, Dr. S. Periyalwar, Carleton University, January, 2005 (available at http://www.sce.carleton.ca/faculty/yanikomeroglu/Pub/mubarek_thesis.p df) 
[5] Z. Kostic et al. "Dynamic frequency hopping in cellular systems with network assisted resource allocation," IEEE Vehicular Technology

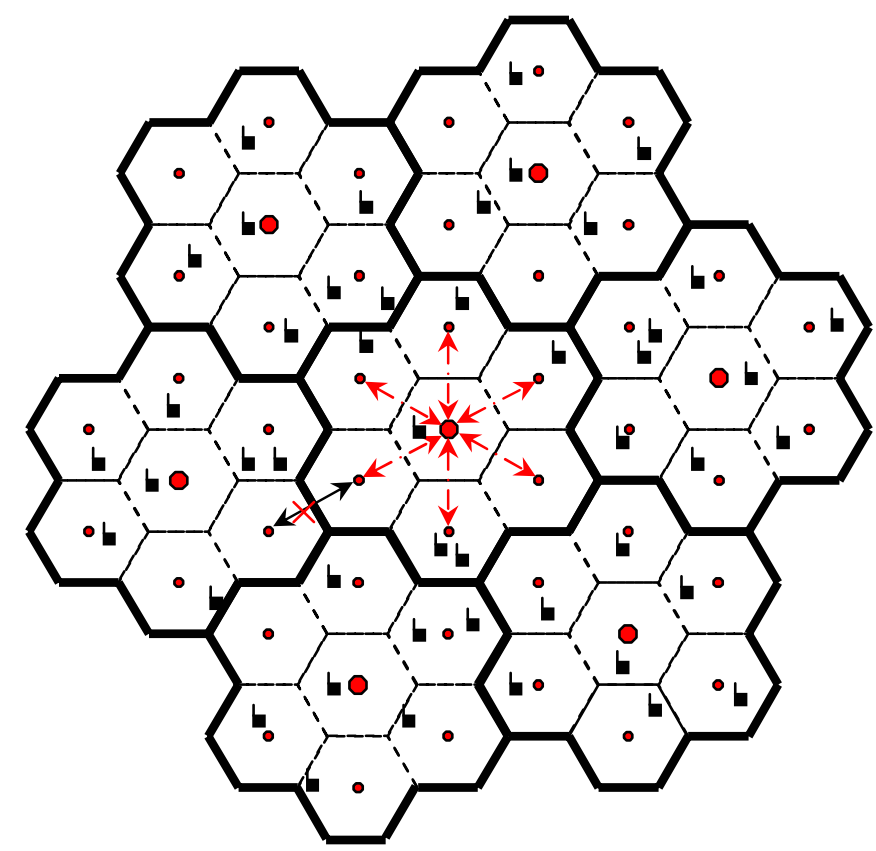

Fig. 1 System architecture for the proposed relay system
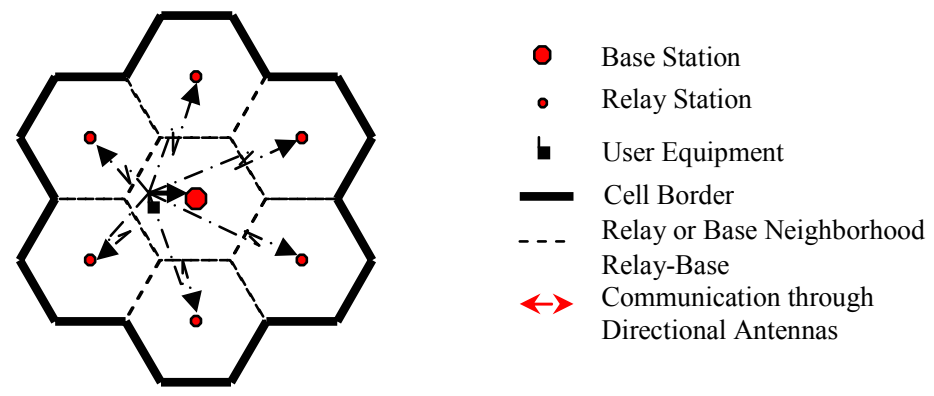

Fig. 2 BS - UE communication

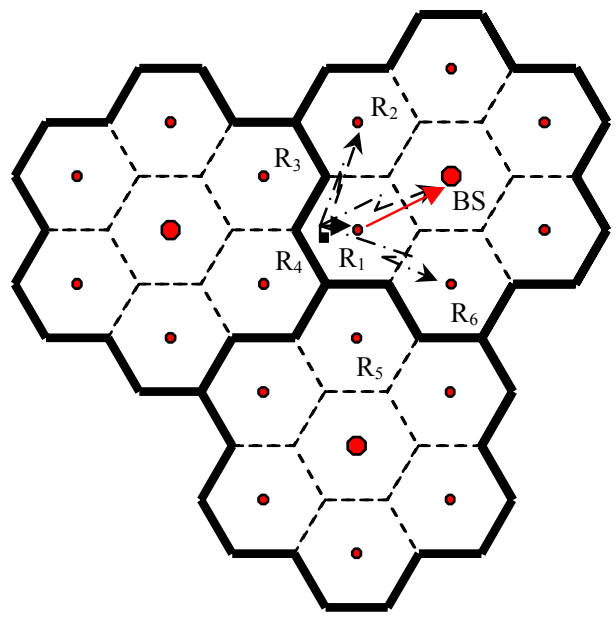

Fig. 3 RS - UE communication
Conference Spring 2000 (VTC'S00), vol.3, pp..2459-2463, Tokyo, Japan.

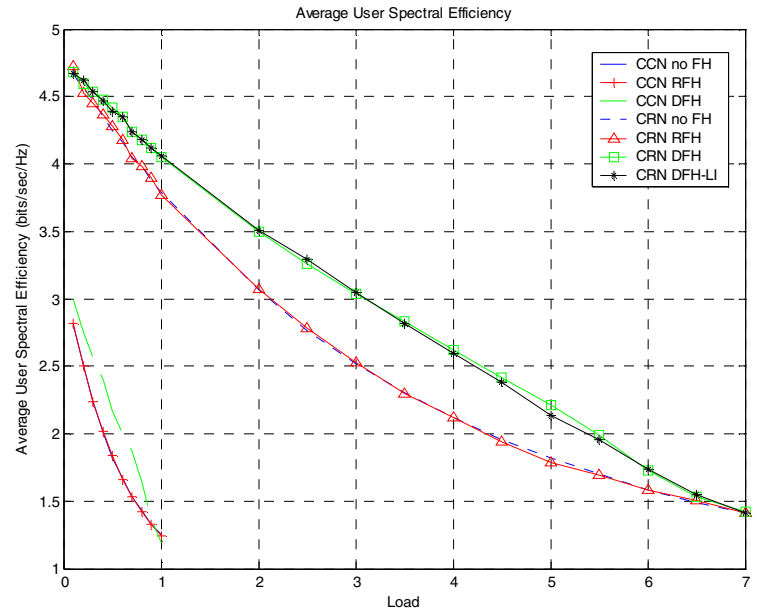

Fig. 4 Average user spectral efficiency

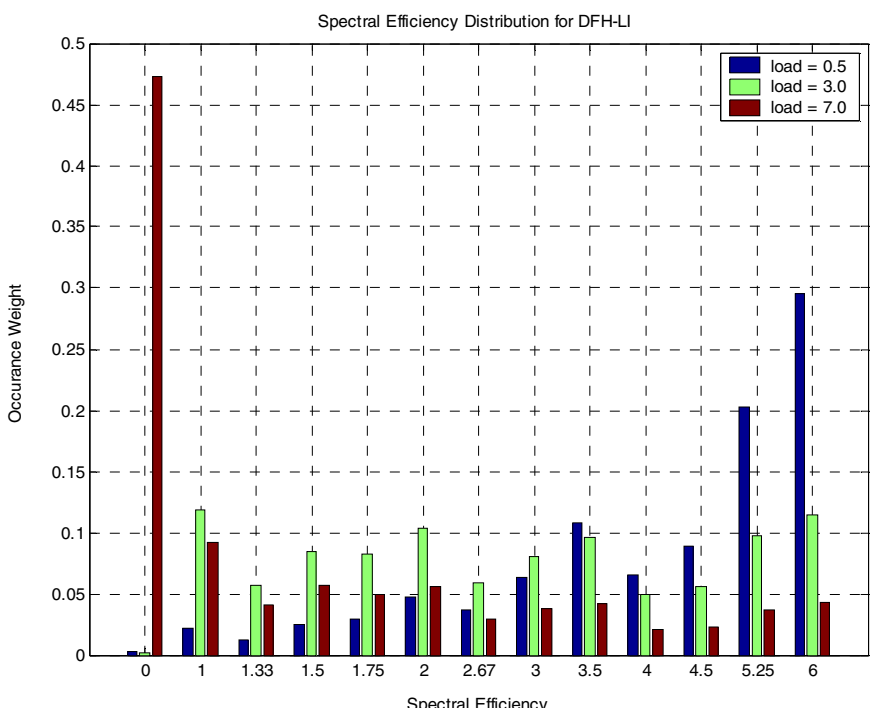

Fig. 5 Average poor FH ratio over the whole FH pattern

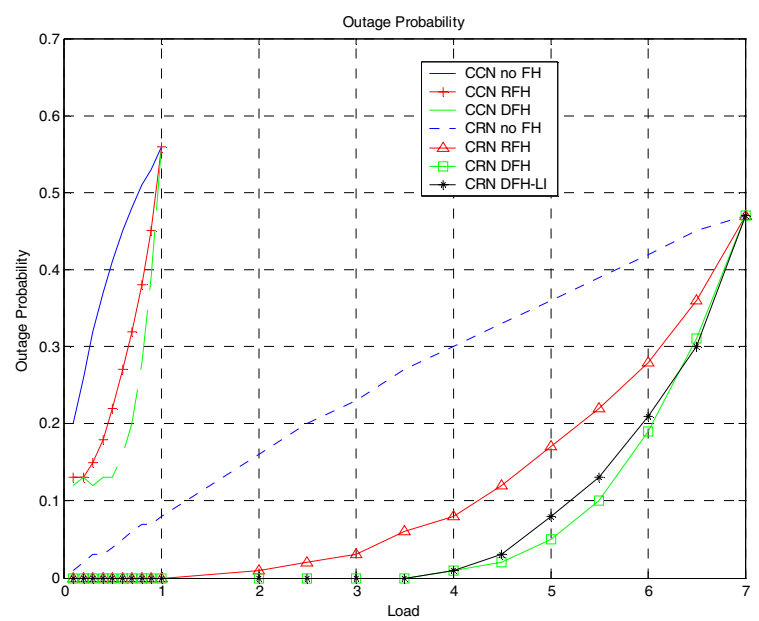

Fig. 6 Outage probability 